\title{
Vibrational Action Spectroscopy of Solids: New Surface-Sensitive Technique
}

\author{
Zongfang Wu, ${ }^{1}$ Agata Płucienik, ${ }_{1}^{1}$ Felix E. Feiten, ${ }^{1,}{ }^{*}$ Matthias Naschitzki, ${ }^{1}$ Walter Wachsmann, ${ }^{1}$ Sandy Gewinner, ${ }^{1}$ \\ Wieland Schöllkopf, ${ }^{1}$ Volker Staemmler, ${ }^{2}$ Helmut Kuhlenbeck, ${ }^{1}$ and Hans-Joachim Freund ${ }^{1}$ \\ ${ }^{1}$ Fritz Haber Institute of the Max Planck Society, Faradayweg 4-6, 14195 Berlin, Germany \\ ${ }^{2}$ Ruhr-Universität Bochum, Lehrstuhl für Theoretische Chemie, Universitätsstraße 150, 44801 Bochum, Germany
}

(Received 13 June 2017; published 28 September 2017)

\begin{abstract}
Vibrational action spectroscopy employing infrared radiation from a free-electron laser has been successfully used for many years to study the vibrational and structural properties of gas phase aggregates. Despite the high sensitivity of this method no relevant studies have yet been conducted for solid sample surfaces. We have set up an experiment for the application of this method to such targets, using infrared light from the free-electron laser of the Fritz Haber Institute. In this Letter, we present first results of this technique with adsorbed argon and neon atoms as messengers. We were able to detect surface-located vibrations of a thin $\mathrm{V}_{2} \mathrm{O}_{3}(0001)$ film on $\mathrm{Au}(111)$ as well as adsorbate vibrations, demonstrating that this method is highly surface sensitive. We consider that the dominant channel for desorption of the messenger atoms is direct inharmonic vibrational coupling, which is essentially insensitive to subsurface or bulk vibrations. Another channel is thermal desorption due to sample heating by absorption of infrared light. The high surface sensitivity of the nonthermal channel and its insensitivity to subsurface modes makes this technique an ideal tool for the study of surface-located vibrations.
\end{abstract}

DOI: 10.1103/PhysRevLett.119.136101

Vibrational spectroscopy provides key information on surfaces and the interaction of adsorbed species with those surfaces. The most prominent examples are infrared reflection absorption spectroscopy (IRAS) (for some early examples see Refs. [1-3]) and high-resolution electron energy loss spectroscopy (HREELS) [4]. Both techniques exhibit considerable drawbacks. In IRAS, the intense instrumental contributions to the spectrum are removed via division by a reference sample spectrum, which has the consequence that an IRAS spectrum contains structures not only of the sample under consideration, but also of the reference sample, which can cause ambiguities [5]. In HREELS spectra of oxides or other ionic compounds, surface related species and objects are buried under the dominant substrate phonons and the spectra need additional data treatment to reveal the desired information. We present here the first result using a technique that does not suffer from those drawbacks: action spectroscopy of surfaces using rare gas messenger atoms.

The method, often used to study gas phase aggregates, [6-20] is based on the idea that rare gas atoms bound to an aggregate at low temperature will desorb when infrared (IR) radiation in resonance with a vibration of the aggregate heats it up. Thus, a plot of the rare gas desorption rate-recorded with a mass spectrometer-vs the IR photon energy represents a vibrational spectrum (see Refs. $[21,22]$ for an overview). This Letter reports the first successful application of this technique to solid surfaces, i.e., a thin $\mathrm{V}_{2} \mathrm{O}_{3}(0001)$ oxide layer on $\mathrm{Au}(111)$ and a $\mathrm{TiO}_{2}(110)$ single crystal.

We have constructed an apparatus for this experimental technique, which uses the tunable infrared radiation from the free-electron laser (FEL) at the Fritz Haber Institute
[23]. The essential components of this setup are a highly sensitive quadrupole mass spectrometer (QMS) in a separately pumped housing and a helium cryostat for sample cooling. The FEL light is focused onto a sample area with a diameter of roughly $3 \mathrm{~mm}$ at an incidence angle of $70^{\circ}$ relative to the surface normal with the sample being positioned directly in front of the entrance opening of the QMS housing (see Fig. 1). Vibrational action spectra are recorded by the messenger atom method [24], employing adsorbed argon or neon.

The Fritz Haber FEL generates macropulses (5-8 $\mu \mathrm{s}$ long pulse trains at $10 \mathrm{~Hz}$ repetition rate) consisting of some thousand micropulses at a rate of $1 \mathrm{GHz}$, each of them a few psec long [23]. The energy per micropulse is close to $10 \mu \mathrm{J}$ corresponding to $\sim 50-80 \mathrm{~mJ}$ per macropulse. The spectral width of the radiation is about $0.5 \%$ (full width at half maximum) of its central wavelength [23].

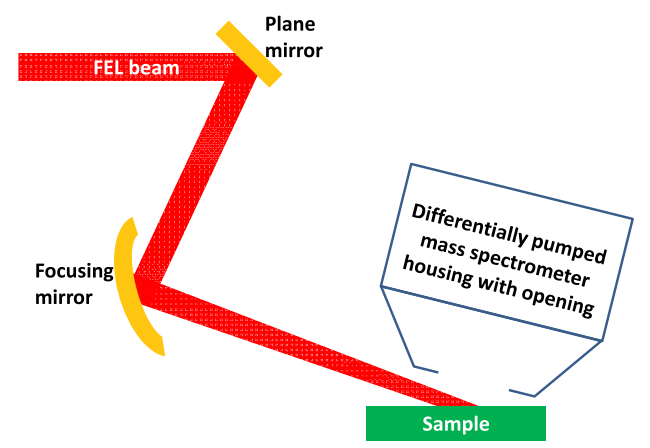

FIG. 1. Scheme of the experimental setup for vibrational action spectroscopy of surfaces. 
A $\mathrm{V}_{2} \mathrm{O}_{3}(0001)$ film $(\sim 100 \AA$ thick $)$ on $\mathrm{Au}(111)$ was chosen as the initial test system since the surface is terminated by vanadyl $(\mathrm{V}=\mathrm{O})$ groups, which are not found in the bulk. This has the advantage that their vibration can be used to check the surface sensitivity of the method. The $\mathrm{V}=\mathrm{O}$ stretching mode has an energy of $\sim 1040 \mathrm{~cm}^{-1}$ as determined with HREELS and IRAS [25-29], with the energy varying somewhat between different samples. STM and IV-LEED have independently verified the presence of these groups at the surface [30,31].

$\mathrm{The} \mathrm{Au}(111)$ substrate also has profitable properties. Gold has the highest midinfrared reflectivity among all metals, which has two advantages: due to the low IR absorption cross section (and the high thermal conductivity) the sample warming up during the experiments will be small and the IR absorption of the oxide layer will be high due to the increased IR intensity at the surface [32]. The $\mathrm{V}_{2} \mathrm{O}_{3}(0001)$ film was prepared on clean $\mathrm{Au}(111)$ following a previously reported recipe [27,28,31].

A set of data of $\mathrm{V}_{2} \mathrm{O}_{3}(0001) / \mathrm{Au}(111)$ is shown in Fig. 2. Curve (a) displays an IRAS trace representing a spectrum of vanadyl terminated $\mathrm{V}_{2} \mathrm{O}_{3}(0001)$ divided by a spectrum of $\mathrm{V}_{2} \mathrm{O}_{3}(0001)$ with most of the vanadyl groups

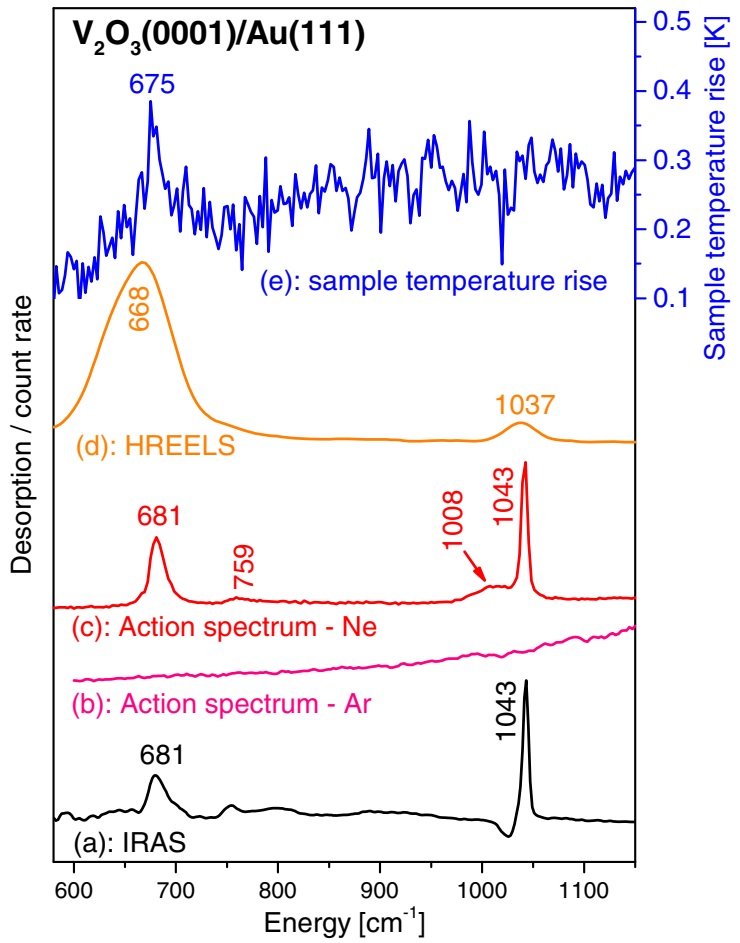

FIG. 2. Comparison of different spectra of $\mathrm{V}_{2} \mathrm{O}_{3}(0001) /$ $\mathrm{Au}(111)$. Spectra (b) and (c) were measured from high to low energy with the slowly decreasing background intensity being mostly due to desorption of rare gas from the cryostat and the sample holder. Spectrum (e) is the sample temperature rise for spectrum (c) relative to the sample temperature before infrared irradiation. The scale on the right ordinate axis applies to curve (e) only. removed. Some remnants of the vanadyl layer on the latter surface lead to the minimum at $\sim 1020 \mathrm{~cm}^{-1}$ (the vibrational energy of vanadyl groups depends to some extent on preparation and coverage.). The bulk-related states are removed by the division procedure so that the bands in the IRAS spectrum are related exclusively to the surfaces of both the sample and the reference sample. In contrast to IRAS, HREELS is also sensitive to subsurface modes. Bulk-related intensity is expected between 600 and $700 \mathrm{~cm}^{-1}$ [33,34], which is the reason why the HREELS spectrum (d) exhibits much more intensity in this range than the IRAS spectrum. The vanadyl vibration at $\sim 1040 \mathrm{~cm}^{-1}$ is surface related, and there are no bulk states expected in this energy range. Spectra labeled (b) and (c) in Fig. 2 are action spectra using argon and neon as messenger gases, respectively. Apparently, only the neon messenger works. There is no signal above background for argon. The action spectrum (c) is very similar to the IRAS spectrum (a), while it is significantly different from the HREELS spectrum (d) between 600 and $700 \mathrm{~cm}^{-1}$. The reason is probably that this energy range contains bulk as well as surface states. HREELS detects surface and bulk state related intensity while IRAS and action spectroscopy are sensitive to the surface related component(s) only.

Previously published STM data show that missing vanadyl groups are common defects on the $\mathrm{V}_{2} \mathrm{O}_{3}(0001)$ surface. Sometimes also not well-characterized protrusions are seen [31]. The mode at $1008 \mathrm{~cm}^{-1}$ may be related to vanadyl groups near to such defects and the mode at $759 \mathrm{~cm}^{-1}$ is probably also related to surface defects since bulk modes are not expected at this energy $[33,34]$.

A careful thermal desorption study by Schlichting and Menzel reports activation energies in the range of 220 and $830 \mathrm{~cm}^{-1}$ for neon and argon desorption from $\mathrm{Ru}(0001)$, respectively $[35,36]$. Schlichting compiles activation energies also for a number of other substrates in his thesis (Ref. [35], tables V.I.20 and V.I.21), with the values ranging from $\sim 81$ to $\sim 1000 \mathrm{~cm}^{-1}$ for neon and from $\sim 660$ to $\sim 960 \mathrm{~cm}^{-1}$ for argon. The upper limit for neon is an exceptionally high singular value. If this value is not considered then the upper limit is $229 \mathrm{~cm}^{-1}$.

We have performed preliminary quantum chemical calculations of the binding energies of neon and argon to the vanadyl groups for linear $\mathrm{V}=\mathrm{O}-\mathrm{Rg}(\mathrm{Rg}=\mathrm{Ne}, \mathrm{Ar})$ configurations. An approximate coupled cluster with singles and doubles method [37] with a reasonably large basis set was applied. The vanadyl groups at the $\mathrm{V}_{2} \mathrm{O}_{3}(0001)$ surface were modeled in two ways, first by an isolated $\mathrm{V}_{2} \mathrm{O}_{5}$ molecule with a symmetric surfacelike structure $\mathrm{O}=\mathrm{V}-$ $\mathrm{O}_{3}-\mathrm{V}=\mathrm{O}$ and then by a neutral $\mathrm{V}_{2} \mathrm{O}_{14}$ cluster with the structure $\mathrm{O}=\mathrm{V}-\mathrm{O}_{6}-\mathrm{V}_{3}-\mathrm{V}_{3}-\mathrm{O}_{6}-\mathrm{V}=\mathrm{O}$. See Supplemental Material [38] for the structure of the clusters and some details of the calculations. The binding energies $D_{0}$ for neon and argon on the $\mathrm{V}_{2} \mathrm{O}_{5}$ molecule were calculated to 22 and $47 \mathrm{~cm}^{-1}$, respectively. They are slightly smaller, 16 
and $38 \mathrm{~cm}^{-1}$, on the $\mathrm{V}_{8} \mathrm{O}_{14}$ surface cluster. The true binding energies are certainly larger since the $\mathrm{V}=\mathrm{O}-\mathrm{Rg}$ bonding is dominated by van der Waals forces which are underestimated by our method and basis set. However, since the polarizability of oxygen in metal oxides is similar to that of neon, we are confident that the error of our results is not larger than $30 \%-40 \%$ (the dissociation energy of $\mathrm{Ne}-\mathrm{Ne}$ is $16.5 \mathrm{~cm}^{-1}$ [39], that of Ne-Ar $32.9 \mathrm{~cm}^{-1}$ [40]). These values are significantly smaller than the values reported by Schlichting, which is probably to a large part due to the absence of lateral adsorbate interactions in the cluster models. In a hexagonal layer each atom would interact with six neighbors, which would contribute significantly to the binding energies. Another aspect is that the rare gas adsorption sites might be different from the ones assumed in the cluster models. However, finding the right adsorption site would require to theoretically model an adsorbate layer, which was beyond the scope of the present study. Finally, we mention that the energies derived by thermal desorption spectroscopy are activation energies while the computed values are true binding energies. Thus, the theoretical results essentially reflect the on-top bonding of a single atom to a vanadyl group, while the thermal desorption results are activation energies for the removal of atoms out of adsorbate layers on $\mathrm{Ru}(0001)$.

Nevertheless, both theoretical and experimental results clearly show that desorbing Ar atoms from the surface requires significantly more energy than desorbing $\mathrm{Ne}$ atoms. In view of this, the experimental result that argon atoms could not be desorbed by the FEL IR irradiation while neon atoms could be desorbed may be explained such that the energy transferred into the rare gas atom bonds to the surface and to neighboring rare gas atoms was high enough to desorb neon but not high enough for argon. We note that for vibrations with an energy lower than the activation energy for desorption, desorption gets more and more unlikely with decreasing vibrational energy if the energy is between the activation energy and the binding energy $D_{0}$, and it gets impossible for vibrational energies below $D_{0}$.

A likely energy transfer mechanism is the inharmonic coupling between vibrational levels. After resonant absorption of a photon, the $\mathrm{V}=\mathrm{O}$ vibration is excited. Because of the coupling of the vibrational states in the system, energy may be transferred to the $\mathrm{O}-\mathrm{Ne}$ oscillator and other vibrations, which compete with the $\mathrm{O}-\mathrm{Ne}$ vibration for energy. The neon atoms may eventually desorb if a sufficient amount of energy is transferred to the O-Ne bond. This energy transfer mechanism will only be effective if the vibrational wave function of the mode excited by the laser light overlaps with the wave function of the neon vs surface vibration. This requirement causes the observed high sensitivity of the method to vibrational levels at the surface and its insensitivity to vibrations below the surface.

As an additional test of surface sensitivity, the method was applied to adsorbate covered $\mathrm{V}_{2} \mathrm{O}_{3}(0001)$. Methanol

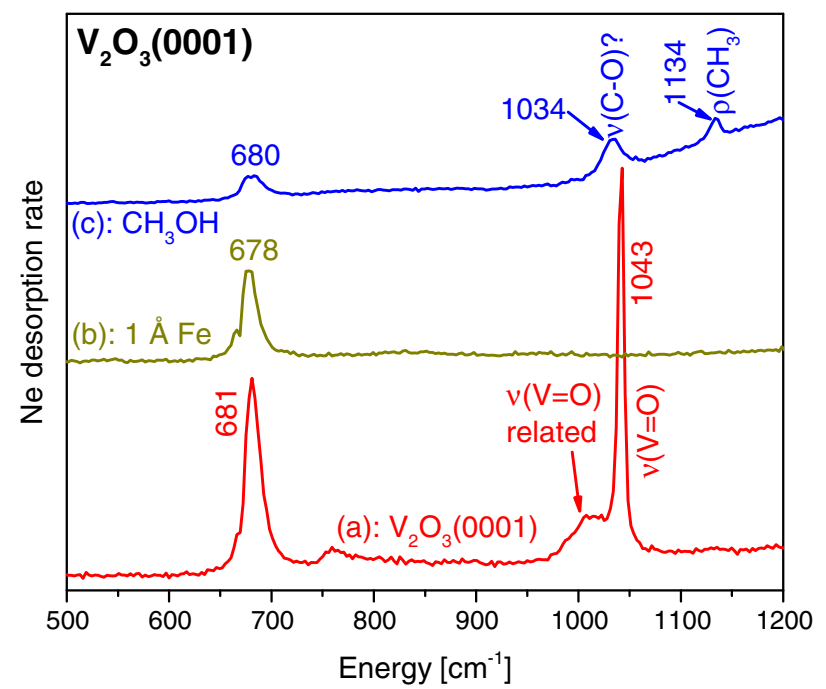

FIG. 3. Action spectra with neon as messenger gas of (a) vanadyl-terminated $\mathrm{V}_{2} \mathrm{O}_{3}(0001) / \mathrm{Au}(111)$, (b) $1 \AA$ iron on $\mathrm{V}_{2} \mathrm{O}_{3}(0001) / \mathrm{Au}(111)$ (deposited at $300 \mathrm{~K}$ ), and (c) methanol on $\mathrm{V}_{2} \mathrm{O}_{3}(0001) / \mathrm{Au}(111)$ (dosed at $8 \mathrm{~K}$ followed by a flash to $200 \mathrm{~K}$ to remove methanol above the first layer).

$\left(\mathrm{CH}_{3} \mathrm{OH}\right)$ was chosen as the adsorbate for the test experiment since it has been studied in detail in preceding experiments $[41,42] . \mathrm{CH}_{3} \mathrm{OH}$ was dosed at $8 \mathrm{~K}( \pm 3 \mathrm{~K})$ onto the sample, followed by a flash to $200 \mathrm{~K}$ to remove the methanol layers above the first layer. Ne action spectra are shown in Fig. 3. The modes of the clean surface are missing or strongly attenuated in the spectrum of the methanol covered surface [Fig. 3(c)] and a peak related to the $\mathrm{CH}_{3}$ rocking mode of methanol shows up at $1134 \mathrm{~cm}^{-1}$. The peak at $1034 \mathrm{~cm}^{-1}$ may be attributed to the methanol C-O vibration, but we cannot exclude that it contains remaining vanadyl intensity. Figure 3 also shows a spectrum obtained after deposition of $1 \AA$ of iron onto $\mathrm{V}_{2} \mathrm{O}_{3}(0001)$. In this spectrum the vanadyl mode is not detected any more, while the peak at $681 \mathrm{~cm}^{-1}$ is attenuated to half of its intensity, which may be attributed to an incomplete iron surface coverage, since $1 \AA$ of iron is less than half a monolayer. These results fully support the conclusion that the action spectroscopy method is a highly surface sensitive technique.

Figure 2(e) shows that the sample was warmed up by the infrared radiation in the energy region associated with the $\mathrm{V}_{2} \mathrm{O}_{3}$ bulk states. Because of the limited thickness of the $\mathrm{V}_{2} \mathrm{O}_{3}(0001)$ layer and to the presence of the gold single crystal substrate with its high thermal conductivity, the temperature rise was limited to $0.38 \mathrm{~K}$ for $\mathrm{V}_{2} \mathrm{O}_{3}(0001)$ / $\mathrm{Au}(111)$, which probably did not induce thermal desorption of neon.

Nevertheless, there may also be a thermal desorption channel related to sample warming. Sample warm-up due to excitation of bulk polaritons is more prominent for oxide single crystals than for thin oxide layers on thermally wellconductive metal single crystals. Effects related to this were 


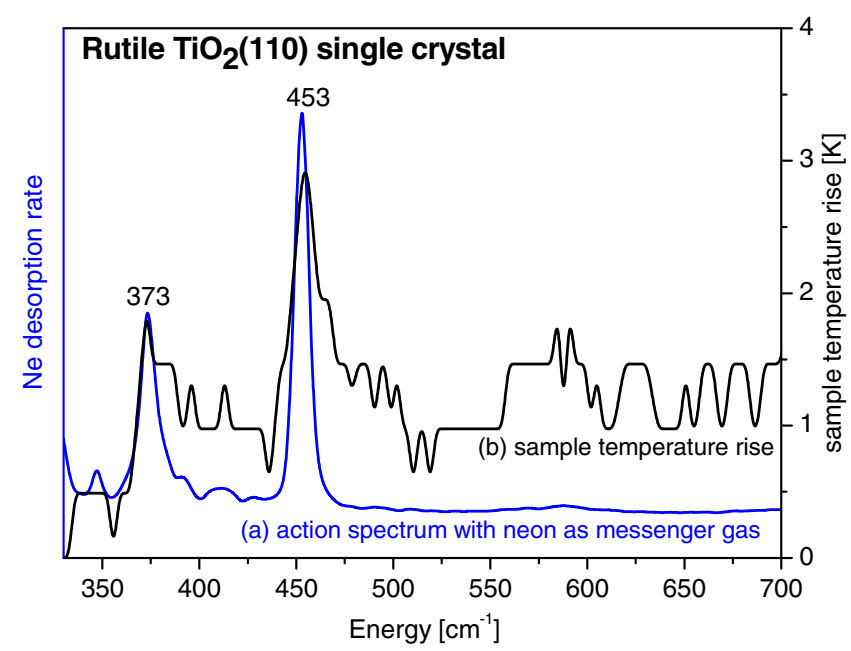

FIG. 4. (a) Action spectrum of rutile $\mathrm{TiO}_{2}(110)$ with neon as messenger gas, (b) sample temperature rise.

studied for a rutile $\mathrm{TiO}_{2}(110)$ single crystal. Figure 4 displays a Ne action spectrum. Henderson [43] found levels at 365 and $445 \mathrm{~cm}^{-1}$ using HREELS, which probably correspond to the states observed at 373 and $453 \mathrm{~cm}^{-1}$ in Fig. 4.

In the $\mathrm{TiO}_{2}(110)$ case, the sample temperature rise $(\sim 3 \mathrm{~K})$ is significantly higher than that observed for $\mathrm{V}_{2} \mathrm{O}_{3}(0001)$ on $\mathrm{Au}(111)(0.38 \mathrm{~K})$. Neon desorption occurs below $\sim 12 \mathrm{~K}$ for $\mathrm{Ru}(0001)$ [36] and one may expect that the temperature is not very much different for rutile $\mathrm{TiO}_{2}(110)$. The sample temperature is not accurately known in the present case due to the use of a type $\mathrm{K}$ thermocouple, which is not very accurate at these low temperatures, but it is well possible that a temperature increase of $3 \mathrm{~K}$ induces thermal desorption. The transient temperatures at the sample surface may even be higher since the FEL beam consists of short intense pulses [23] and the thermal conductivity of $\mathrm{TiO}_{2}$ is limited. The shape of the desorption peaks is fairly symmetric, somewhat different from the shape of the temperature peaks which might indicate that even in this case a direct inharmonic vibrational energy transfer drives the rare gas desorption, but it may also mean that the transient surface temperature maxima cause it. We note that thermal effects can be reduced. One handle towards this is lowering of the FEL power, especially in combination with the use of supported thin film samples due to the better heat conductivity. A large illuminated sample area may also help to reduce local heating. In any case, a good thermal contact of the sample to the cryostat will be profitable.

In the following we will dwell on the desorption mechanism in the nonthermal channel. The vibrational energy will thermalize in the time span between two FEL micropulses $(1 \mathrm{nsec})$ and is not available anymore for neon desorption when the next micropulse arrives. We note that this statement is not completely safe since there are systems for which it does not apply [44,45], but for the vanadyl case it does likely apply, since the energies of the substrate vibrations and the vanadyl vibration are not vastly different and the substrate-vanadyl coupling is not weak.

With a FEL micropulse energy of $10 \mu \mathrm{J}$, an infrared absorption by the vanadyl groups of $\sim 1 \%$ as derived from the infrared spectrum in Fig. 2(a), an illuminated sample area with a diameter of $3 \mathrm{~mm}$, and one vanadyl group per unit cell at the surface [31], it can be estimated that one vanadyl group absorbs on average $\sim 0.1$ photons per micropulse. Variation of the size of the illuminated area and variation of the beam intensity did not provide clear evidence for a nonlinear dependence of the desorption rate on the beam intensity, which means that according to present knowledge, desorption is probably triggered by absorption of a single photon. If every absorbed photon would lead to desorption then essentially all rare gas atoms would be desorbed after a single macropulse. This is not in agreement with the experimental results, demonstrating that only a small fraction of the absorbed photons actually induces desorption. In the case of argon desorption the desorption energy is higher and therefore the fraction of photons which lead to desorption will be even smaller, too small for the detection of desorbing argon atoms in the vanadyl case.

In summary, we have shown that the vibrational messenger atom action spectroscopy can be applied to surfaces. Because of the high surface sensitivity of the technique it can be applied to a wide range of topics in model catalysis and general surface science. In contrast to IRAS this method does not require reference spectra, and in contrast to HREELS it does not suffer from multiple excitations. The method is not only surface sensitive, it is probably also insensitive to subsurface vibrations if thermal desorption can be avoided. One yet untouched aspect is that it is possible to prepare rare gas layers on inhomogeneous surfaces such that only areas with high adsorption energies are covered. This can be achieved by dosing a submonolayer amount of rare gas or by choosing the adsorption temperature such that sites with a low rare gas binding energy cannot be populated. If the populated sites are sites on a cluster then the signal would stem exclusively from them. Such a selective cluster-sensitive spectroscopy might have a significant impact on the area of model catalysis.

The Fonds der Chemischen Industrie is gratefully acknowledged for financial support. Z.W. thanks the Alexander von Humboldt Foundation (AvH) for support.

* Present address: Hokkaido University, Institute for Catalysis, Kita21, Nishi10, Kita-ku, Sapporo, Hokkaido, Japan, 001-0021.

[1] B. E. Hayden and A. M. Bradshaw, Surf. Sci. 125, 787 (1983). 
[2] A. M. Bradshaw and F. M. Hoffmann, Surf. Sci. 72, 513 (1978).

[3] H. Pfnür, D. Menzel, F. M. Hoffmann, A. Ortega, and A. M. Bradshaw, Surf. Sci. 93, 431 (1980).

[4] H. Ibach and D. L. Mills, Electron Energy Loss Spectroscopy and Surface Vibrations (Academic Press, New York, 1982).

[5] M. K. Ainsworth, M. R. S. McCoustra, M. A. Chesters, N. Sheppard, and C. De La Cruz, Surf. Sci. 437, 9 (1999).

[6] B. Simard, S. Dénommée, D. M. Rayner, D. v. Heijnsbergen, G. Meijer, and G. v. Helden, Chem. Phys. Lett. 357, 195 (2002).

[7] M. Brümmer, C. Kaposta, G. Santambrogio, and K. R. Asmis, J. Chem. Phys. 119, 12700 (2003).

[8] A. Fielicke, G. Meijer, and G. von Helden, J. Am. Chem. Soc. 125, 3659 (2003).

[9] D. van Heijnsbergen, K. Demyk, M. A. Duncan, G. Meijer, and G. von Helden, Phys. Chem. Chem. Phys. 5, 2515 (2003).

[10] A. Fielicke, A. Kirilyuk, C. Ratsch, J. Behler, M. Scheffler, G. von Helden, and G. Meijer, Phys. Rev. Lett. 93, 023401 (2004).

[11] A. Fielicke, C. Ratsch, G. von Helden, and G. Meijer, J. Chem. Phys. 122, 091105 (2005).

[12] A. Fielicke, G. von Helden, G. Meijer, B. Simard, and D. M. Rayner, J. Phys. Chem. B 109, 23935 (2005).

[13] P. Gruene, D. M. Rayner, B. Redlich, A. F. G. van der Meer, J. T. Lyon, G. Meijer, and A. Fielicke, Science 321, 674 (2008).

[14] I. Swart, F. M. F. de Groot, B. M. Weckhuysen, P. Gruene, G. Meijer, and A. Fielicke, J. Phys. Chem. A 112, 1139 (2008).

[15] R. Gehrke, P. Gruene, A. Fielicke, G. Meijer, and K. Reuter, J. Chem. Phys. 130, 034306 (2009).

[16] L. Lin et al., Phys. Chem. Chem. Phys. 12, 13907 (2010).

[17] M. Haertelt, V. J. F. Lapoutre, J. M. Bakker, B. Redlich, D. J. Harding, A. Fielicke, and G. Meijer, J. Phys. Chem. Lett. 2, 1720 (2011).

[18] A. P. Woodham, G. Meijer, and A. Fielicke, Angew. Chem., Int. Ed. 51, 4444 (2012).

[19] A. P. Woodham, G. Meijer, and A. Fielicke, J. Am. Chem. Soc. 135, 1727 (2013).

[20] M. R. Fagiani, X. Song, P. Petkov, S. Debnath, S. Gewinner, W. Schöllkopf, T. Heine, A. Fielicke, and K. R. Asmis, Angew. Chem., Int. Ed. 56, 501 (2017).

[21] K. R. Asmis, Phys. Chem. Chem. Phys. 14, 9270 (2012).

[22] A. M. Rijs and J. Oomens, in Gas-Phase IR Spectroscopy and Structure of Biological Molecules, edited by A. M. Rijs, and J. Oomens, (Springer International Publishing, Switzerland, 2015), p. 1.

[23] W. Schöllkopf, S. Gewinner, H. Junkes, A. Paarmann, G. von Helden, H. Bluem, and A. M. M. Todd, Proc. SPIE Int. Soc. Opt. Eng. 9512, 95121L (2015).

[24] A. Fielicke, G. von Helden, and G. Meijer, Eur. Phys. J. D 34, 83 (2005).
[25] F. Pfuner, J. Schoiswohl, M. Sock, S. Surnev, M. G. Ramsey, and F. P. Netzer, J. Phys. Condens. Matter 17, 4035 (2005).

[26] J. Schoiswohl, G. Tzvetkov, F. Pfuner, M. G. Ramsey, S. Surnev, and F. P. Netzer, Phys. Chem. Chem. Phys. 8, 1614 (2006).

[27] A. C. Dupuis, M. Abu Haija, B. Richter, H. Kuhlenbeck, and H. J. Freund, Surf. Sci. 539, 99 (2003).

[28] M. A. Haija, Doctoral Thesis, Technische Universität Berlin, Fakultät II - Mathematik und Naturwissenschaften, 2006.

[29] M. A. Haija, Y. Romanyshyn, A. Uhl, H. Kuhlenbeck, and H.-J. Freund, Top. Catal. 60, 413 (2017).

[30] F. E. Feiten, J. Seifert, J. Paier, H. Kuhlenbeck, H. Winter, J. Sauer, and H.-J. Freund, Phys. Rev. Lett. 114, 216101 (2015).

[31] F. E. Feiten, H. Kuhlenbeck, and H.-J. Freund, J. Phys. Chem. C 119, 22961 (2015).

[32] J. T. Yates and C. J. Powell, Methods of surface characterization, in Vibrational Spectroscopy of Molecules on Surfaces (Springer Science+Business Media, New York, 1978).

[33] G. Kresse, S. Surnev, J. Schoiswohl, and F. P. Netzer, Surf. Sci. 555, 118 (2004).

[34] M. A. Haija, S. Guimond, Y. Romanyshyn, A. Uhl, H. Kuhlenbeck, T. K. Todorova, M. V. Ganduglia-Pirovano, J. Döbler, J. Sauer, and H.-J. Freund, Surf. Sci. 600, 1497 (2006).

[35] H. Schlichting, Ph.D. thesis, Technische Universität München, 1990.

[36] H. Schlichting and D. Menzel, Rev. Sci. Instrum. 64, 2013 (1993).

[37] R. Fink and V. Staemmler, Theor. Chim. Acta 87, 129 (1993).

[38] See Supplemental Material at http://link.aps.org/ supplemental/10.1103/PhysRevLett.119.136101 for details of the calculations.

[39] R. A. Aziz and M. J. Slaman, Chem. Phys. 130, 187 (1989).

[40] J. U. Grabow, A. S. Pine, G. T. Fraser, F. J. Lovas, R. D. Suenram, T. Emilsson, E. Arunan, and H. S. Gutowsky, J. Chem. Phys. 102, 1181 (1995).

[41] Y. Romanyshyn, S. Guimond, D. Göbke, J. M. Sturm, H. Kuhlenbeck, J. Döbler, M. V. Ganduglia-Pirovano, J. Sauer, and H. J. Freund, Top. Catal. 54, 669 (2011).

[42] D. Göbke, Y. Romanyshyn, S. Guimond, J. M. Sturm, H. Kuhlenbeck, J. Döbler, U. Reinhardt, M. V. GandugliaPirovano, J. Sauer, and H.-J. Freund, Angew. Chem., Int. Ed. 48, 3695 (2009).

[43] M. A. Henderson, Surf. Sci. 355, 151 (1996).

[44] H. Gai and G. A. Voth, J. Chem. Phys. 99, 740 (1993).

[45] H. Xu, L. Kristian, and H. Eckart, J. Phys. Condens. Matter 20, 224008 (2008). 